OPEN ACCESS

Edited by:

Junjie Xiao,

Shanghai University, China

Reviewed by:

Atsuhiko Yagishita,

Tokai University Isehara

Hospital, Japan

Nazareno Paolocci,

Johns Hopkins University,

United States

Zhengyuan Xia,

The University of Hong Kong,

Hong Kong

Gaetano Ruocco,

Regina Montis Regalis Hospital, Italy

Valdo Jose Dias Da Silva

Universidade Federal do Triângulo

Mineiro, Brazil

${ }^{*}$ Correspondence:

Marvin Wei Jie Chua

marvin.chua.w.j@singhealth.com.sg

Specialty section:

This article was submitted to

General Cardiovascular Medicine,

a section of the journal

Frontiers in Cardiovascular Medicine

Received: 06 July 2020 Accepted: 28 September 2020

Published: 30 October 2020

Citation:

Chua MWJ and Mok SF (2020) Amiodarone Induced Thyrotoxicosis and Treatment Complications in a Man With Cyanotic Congenital Heart

Disease: A Case Report.

Front. Cardiovasc. Med. 7:574391

doi: 10.3389/fcvm.2020.574391

\section{Amiodarone Induced Thyrotoxicosis and Treatment Complications in a Man With Cyanotic Congenital Heart Disease: A Case Report}

\author{
Marvin Wei Jie Chua ${ }^{1 *}$ and Shao Feng Mok ${ }^{2}$ \\ ${ }^{1}$ Department of General Medicine, Sengkang General Hospital, Singapore, Singapore, ${ }^{2}$ Division of Endocrinology, \\ Department of Medicine, National University Health System, Singapore, Singapore
}

Background and Case: Amiodarone induced thyrotoxicosis (AIT) is a potentially life-threatening condition that exists in two main subtypes - AIT Type 1 (AIT1) and AIT Type 2 (AIT2). AlT1 is a form of iodine-induced hyperthyroidism with increased thyroid hormone synthesis, while AIT2 is a form of destructive thyroiditis with increased release of pre-formed thyroid hormone. This case report describes a patient with cyanotic congenital heart disease, who developed AIT with severe biochemical thyrotoxicosis. Due to complications to corticosteroids and thionamides, second-line treatment with cholestyramine and lithium was given which eventually restored euthyroidism, averting the need for thyroidectomy and its associated risks. Due to the presence of both typical and unusual features, the final diagnosis of AIT2 could only be retrospectively elucidated after a prolonged clinical course.

Conclusion: Corticosteroids are well-recognized to be the first-line treatment for AIT2. This case illustrates a rare phenomenon: successful treatment of AIT2 with lithium and cholestyramine. In patients who develop complications from first-line therapy, prompt treatment with alternative agents may successfully avert thyroidectomy and its associated risks.

Keywords: amiodarone induced thyrotoxicosis, treatment, complications, cyanotic congenital heart disease, thyrotoxicosis

\section{INTRODUCTION}

Amiodarone induced thyrotoxicosis (AIT) is a potentially catastrophic situation for patients with cardiac disease (1) who are at risk of life-threatening complications. AIT exists in two main subtypes - AIT1 and AIT2, which have different underlying pathophysiological mechanisms (Figure 1). AIT1 is a form of iodine-induced hyperthyroidism caused by the high iodine content of amiodarone, while AIT2 is a form of destructive thyroiditis caused by direct cytotoxic effects of amiodarone and its metabolites.

Diagnosis is often delayed and differentiating between AIT1 and AIT2 is often difficult. 


\section{AlT1}

- lodine-induced hyperthyroidism

- Excess thyroid hormone synthesis due to high iodine content of amiodarone

- Usually occurs in abnormal thyroid glands (eg. latent Graves)

- First-line treatment: thionamides

\section{AIT2}

- Destructive thyroiditis

- Release of pre-formed

Mixed

AIT thyroid hormone due to direct cytotoxic effects of amiodarone

- Usually occurs in normal thyroid glands

- First-line treatment: glucocorticoids

FIGURE 1 | Pathophysiology of various AIT subtypes.

In this case report, we describe the prolonged and tumultuous journey of a young man with cyanotic congenital heart disease who developed AIT. In our bid to urgently reduce serum thyroxine to prevent cardiac decompensation, we encountered significant barriers - the development of complications to first-line therapy, which necessitated the use of secondline agents. It did not help matters that the AIT subtype remained unclear throughout this entire process. Eventually, we managed to successfully restore euthyroidism with lithium and cholestyramine. The successful use of lithium and cholestyramine in the treatment of AIT, let alone AIT2, is rarely reported.

\section{CASE PRESENTATION}

The patient was a 32-year-old gentleman with complex cyanotic congenital cardiac disease consisting of total anomalous venous drainage, double outlet right ventricle and severe pulmonary stenosis, leading to a uni-ventricular circulatory system (ejection fraction was $45 \%$ in April 2017). This was complicated by atrial flutter and complete heart block, which were treated with radiofrequency ablation and permanent pacemaker insertion. Long-term medications included amiodarone which he was taking for the last 3 years due to the recurrence of atrial flutter post-ablation (100 mg daily), digoxin ( $62.5 \mathrm{mcg}$ daily), bisoprolol and warfarin.

The clinical course described spanned 8 months (January to August 2017). Thyroid function test (TFT) monitoring was performed due to long-term amiodarone use. Primary hyperthyroidism was first diagnosed in January 2017, with free thyroxine (fT4) $>72$ (reference interval 8.0-16.0) pmol/L and $\mathrm{TSH}<0.01$ (reference interval 0.45-4.50) $\mathrm{mIU} / \mathrm{L}$ (Table 1), during an admission for lower limb haematoma from warfarin over-anticoagulation. The latter was not a co-incidence, as thyrotoxicosis is known to potentiate the effects of warfarin by increasing the degradation of coagulation factors (2). In addition, pharmacokinetic interaction between amiodarone and warfarin leads to decrease in warfarin requirements, although in our patient amiodarone therapy was long-standing with no changes in dosage (2).

TFTs over the preceding 2 years showed elevated fT 4 with unsuppressed TSH, consistent with inhibition of 5'-deiodinase with decreased deiodination of T4 to triiodothyronine (T3) by amiodarone (3) (Table 1). The marked increase in fT4 over just 3 months is consistent with the well-described sudden and explosive onset of AIT $(1,4)$ (Table $\mathbf{1}$ ).

Apart from mild weight loss, there were no symptoms of thyrotoxicosis, neck swelling or visual symptoms. There was no prior personal or family history of thyroid disease. He was afebrile and hemodynamically stable (blood pressure 126/98 $\mathrm{mmHg}$, pulse rate 83 beats per minute), which was surprising given the degree of biochemical thyrotoxicosis. This could be attributed to in-situ pacemaker with a set rate of 50 beats per minute, as well as chronic beta-blocker and amiodarone therapy. Significant findings included fine hand tremors and a small 
TABLE 1 | TFT Trend while patient was on amiodarone.

\begin{tabular}{lcccccc}
\hline \multirow{2}{*}{\begin{tabular}{l} 
Investigations $\begin{array}{c}\text { Reference } \\
\text { interval }\end{array}$ \\
\cline { 3 - 7 }
\end{tabular}} & & \multicolumn{6}{c}{ Number of months patient was on amiodarone } \\
\cline { 3 - 7 } & & 0 & 3 & 9 & 29 & 32 \\
\hline fT4 (pmol/L) & $8.0-16.0$ & 13.6 & $\mathbf{1 9 . 2}$ & $\mathbf{2 0 . 4}$ & $\mathbf{2 6 . 0}$ & $>\mathbf{7 2 . 0}$ \\
$\mathrm{TSH}(\mathrm{mlU} / \mathrm{L})$ & $0.45-4.50$ & 0.95 & 3.19 & 2.99 & 0.57 & $<\mathbf{0 . 0 1}$ \\
\hline
\end{tabular}

diffuse goiter, but no signs of thyroid eye disease or pretibial myxedema. There was central cyanosis, digital clubbing and a pan-systolic cardiac murmur. His pulse was regular and there were no signs of heart failure.

A provisional diagnosis of AIT was made. Empirical treatment for both subtypes was initiated with carbimazole $40 \mathrm{mg}$ and prednisolone $40 \mathrm{mg}$ daily, while awaiting further investigations to distinguish between AIT type 1 (AIT1) and type 2 (AIT2). Amiodarone was discontinued. Close liver function monitoring was ordered due to carbimazole use in the setting of hyperbilirubinemia (related to hepatic venous congestion). TSH-receptor antibodies and anti-TPO antibodies were negative, while ultrasonography showed thyroid nodules with patchy parenchymal flow. The initial report of the Technetium $\left({ }^{99} \mathrm{Tc}\right)$ pertechnetate scan described diffusely increased thyroid uptake, which suggested AIT1 $(5,6)$. Thus, prednisolone was stopped while carbimazole was continued. However, subsequent development of neutropenia attributed to acute viral illness necessitated carbimazole cessation. Reintroduction of prednisolone at this juncture led to significant reduction in serum thyroxine, but this also had to be discontinued within 1 week due to worsening hepatic dysfunction from acute hepatitis $\mathrm{E}$ infection (Table 2).

The option of total thyroidectomy was considered but abandoned due to prohibitively high surgical and anesthetic risk. Radioactive iodine (RAI) ablation was unlikely to be successful due to low radioactive iodine uptake $(<2 \%)$ after low dose $(100$ micro-CI) I-131 thyroid scan. This was discrepant from the ${ }^{99} \mathrm{Tc}$ scan; a false negative result was unlikely with no recent exposure to iodinated contrast, and thionamides were held off before the scan (7). A possible reason for this discrepancy was that unlike RAI, pertechnetate is rapidly uptaken but not organified. However, when reviewed by another radiologist, interpretation of the ${ }^{99} \mathrm{Tc}$ scan was that of decreased uptake - consistent with the I-131 scan.

Second-line agents - cholestyramine, beta-blockers and lithium, were required to decrease the risk of cardiovascular strain (Table 2). The lack of significant reduction in serum thyroxine despite cholestyramine prompted initiation of propranolol followed by lithium, which was added as a last resort due to its narrow therapeutic index. In our patient, lithium levels were monitored and there were no adverse effects.

Following improvement in hyperbilirubinemia, he was started on thiamazole, which does not require hepatic activation. When he had achieved near-euthyroid status, lithium and cholestyramine were stopped (Table 2). Throughout this entire period, our patient remained stable with no cardiovascular complications.

The patient developed hypothyroidism about 6 months after therapy initiation, and thiamazole was gradually tapered and stopped (Table 2). Post cessation of thiamazole, thyroid function remained normal; but he suffered a recurrence of atrial flutter and supraventricular tachycardia which was treated with nodal radiofrequency ablation. As there were no further arrhythmias, re-initiation of amiodarone was not required.

Throughout this entire journey, we ensured that our patient was closely updated and fully aware of his condition and progress. Before each treatment decision, the risks and benefits were explained in detail, so that an informed decision could be made. For example, before starting lithium, we explained that its utility was to reduce thyroid hormone levels in view of complications to other forms of treatment. However, the potential adverse effects include tremors, diarrhea, polyuria, polydipsia and hypercalcemia (8). Thus, there was a need for close follow up and monitoring of lithium levels. Our patient was adherent to all treatment and follow-up. Indeed, till present, he remains appreciative of our efforts.

\section{DISCUSSION}

AIT is a diagnostic dilemma. Typical clinical features of thyrotoxicosis may be absent because amiodarone leads to decreased catecholamine receptors, decreased T3 effect on betaadrenoceptors and inhibition of T4 to T3 conversion (9), consistent with the observation in our patient. To compound this diagnostic difficulty, management of AIT carries high stakes. AIT is associated with increased morbidity and mortality, and all patients are considered at risk of emergency treatment (10).

Establishing the AIT subtype is often challenging but crucial in determining the optimal treatment strategy (11). Amiodarone is a benzofuranic antiarrythmic drug that contains $\sim 37 \%$ iodine by weight (7). AIT1 is due to excess thyroid hormone synthesis from the high iodine content of amiodarone through the JodBasedow effect $(3,9)$. This usually occurs in patients with preexisting thyroid disease such as latent Graves Disease or nodular goiter, although the role of amiodarone in triggering thyroid autoimmunity is not well established $(1,9)$. First-line treatment is thionamides $(1,12)$, although higher doses and longer treatment are often required due to increased intra-thyroidal iodine load (9). In contrast, AIT2, which usually occurs in normal thyroid glands, is caused by direct cytotoxic effects of amiodarone and/or its metabolite (desethylamiodarone) on thyroid follicular cells, leading to destructive thyroiditis (3). The ideal treatment is glucocorticoids, due to anti-inflammatory and membranestabilizing effects with decreased T4 to T3 conversion (9). Mixed AIT is also recognized, in which both pathogenic mechanisms are implicated and features of both subtypes are often present $(1,7)$.

As our patient had multiple features which supported both AIT1 and AIT2 (Table 3), the AIT subtype was initially unclear, and the possibility of mixed AIT was considered. However, with the benefit of hindsight, the likely diagnosis was AIT2 rather than mixed AIT (Table 3). To begin with, AIT2 is 
TABLE 2 | Summary of clinical course with corresponding treatment.

\begin{tabular}{|c|c|c|c|c|c|c|c|c|c|c|c|c|}
\hline \multirow[t]{2}{*}{ Investigations } & \multirow{2}{*}{$\begin{array}{l}\text { Reference } \\
\text { interval }\end{array}$} & \multicolumn{10}{|c|}{ Number of weeks after diagnosis of amiodarone induced thyrotoxicosis } & \multirow[b]{2}{*}{30} \\
\hline & & 0 & 2 & 3 & 4 & 6 & 7 & 11 & 12 & 15 & 23 & \\
\hline fT4 (pmol/L) & $8.0-16.0$ & $>72.0$ & $>72.0$ & 68.6 & 70.9 & 67.8 & 51.7 & 47.2 & 38.5 & 18.0 & 5.4 & 16.0 \\
\hline $\mathrm{TSH}(\mathrm{mlU} / \mathrm{L})$ & $0.45-4.50$ & $<0.01$ & $<0.01$ & $<0.01$ & $<0.01$ & $<0.01$ & $<0.01$ & $<0.01$ & $<0.01$ & 0.14 & 41.39 & 2.66 \\
\hline Free T3 (pmol/L) & $3.5-6.0$ & & & 11.3 & & & & & & & & \\
\hline Total T3 (nmol/L) & $0.90-2.60$ & & & & & 2.14 & 1.15 & & & & & \\
\hline \multicolumn{13}{|l|}{ Medications } \\
\hline \multicolumn{2}{|l|}{ Carbimazole } & $\sqrt{ }$ & $\sqrt{ }$ & $\sqrt{ }$ & $\sqrt{ }$ & & & & & & & \\
\hline \multicolumn{2}{|l|}{ Thiamazole } & & & & & & & $\sqrt{ }$ & $\sqrt{ }$ & $\sqrt{ }$ & $\sqrt{ }$ & \\
\hline \multicolumn{2}{|l|}{ Bisoprolol } & & $\sqrt{ }$ & $\sqrt{ }$ & $\sqrt{ }$ & $\sqrt{ }$ & $\sqrt{ }$ & $\sqrt{ }$ & $\sqrt{ }$ & $\sqrt{ }$ & $\sqrt{ }$ & $\sqrt{ }$ \\
\hline \multicolumn{2}{|l|}{ Propranolol } & & & & & $\sqrt{ }$ & $\sqrt{ }$ & $\sqrt{ }$ & $\sqrt{ }$ & $\sqrt{ }$ & & \\
\hline \multicolumn{2}{|l|}{ Prednisolone } & $\sqrt{ }$ & & & & $\sqrt{ }$ & & & & & & \\
\hline \multicolumn{2}{|l|}{ Cholestyramine } & & & $\sqrt{ }$ & $\sqrt{ }$ & $\sqrt{ }$ & $\sqrt{ }$ & $\sqrt{ }$ & $\sqrt{ }$ & & & \\
\hline \multicolumn{2}{|l|}{ Lithium } & & & & & & $\sqrt{ }$ & $\sqrt{ }$ & $\sqrt{ }$ & & & \\
\hline \multicolumn{2}{|c|}{ Sequence of events } & & Diagnosis & Viral ne & ropenia & Acut & hepatitis & infection & & Rec & ery & \\
\hline
\end{tabular}

more likely in Singapore, an iodine sufficient region. This diagnosis was supported by the absence of underlying thyroid disease, long duration of prior amiodarone exposure, negative thyroid antibodies and decreased thyroid scan uptake. Despite an apparent initial lack of response, the significant response to corticosteroid reintroduction observed is consistent with a delayed response to corticosteroids in AIT2 patients with markedly elevated thyroid hormone and large thyroid volumes (13), although this could only be given for 1 week due to contraindications. Together with the relatively short duration of thyrotoxicosis and maintenance of euthyroidism without need for further treatment, this further confirmed AIT2.

As outlined above, significant difficulties in management were encountered. This patient developed neutropenia which limited high dose thionamide use, which was not surprising as the risk of carbimazole-induced agranulocytosis is dose-dependent and usually occurs in the first 100 days of therapy (7); as well as acute hepatitis $\mathrm{E}$ infection, which necessitated corticosteroid cessation. At the same time, due to severe biochemical thyrotoxicosis and underlying cyanotic congenital heart disease, urgent reduction of serum thyroxine was essential to decrease the risk of cardiovascular decompensation (7). Thus, thyroidectomy was an important consideration in this patient. In a Mayo clinic case series of 17 AIT patients who underwent thyroidectomy, the indication was severe thyrotoxicosis requiring prompt resolution and intolerance to anti-thyroid drugs in 35 and $24 \%$ of patients, respectively (20); with both of these indications applicable in this patient. Similarly, in another case series, the indication for thyroidectomy in $27 \%$ of patients was side-effects of anti-thyroid drugs (21). The main effect of lithium is inhibition of T4 release (22), while cholestyramine increases enterohepatic clearance of thyroid hormone (23). Although lithium and cholestyramine can be used to treat thyrotoxicosis, reported successful use of these agents in AIT is limited. Lithium has been shown to decrease the time required to achieve euthyroidism in AIT, although not specifically in AIT2 (22). In a case series of 5
AIT2 patients, 2 of them received both cholestyramine and lithium but eventually still required thyroidectomy (24). In our patient, lithium and cholestyramine were crucial in restoring euthyroidism and successfully averting high-risk thyroidectomy.

On top of this, an additional difficulty was the controversial initial thyroid uptake scan report of increased tracer uptake which suggested AIT1, leading to corticosteroids being prematurely stopped after 2 weeks. That said, this was unlikely to have a significant overall impact on management due to subsequent contraindication to corticosteroids.

An important issue we considered was continuation or cessation of amiodarone. Amiodarone is an efficient drug for life-threatening arrythmias, has a long half-life of 90 days and a cardioprotective effect $(9,10,25)$. Thus, stopping amiodarone might increase the risk of life-threatening arrythmias without any short-term benefit $(9,25)$. Although amiodarone continuation might be feasible in AIT2 $[(26,27)$, p. 2], this might lead to increased recurrence rate of thyrotoxicosis and deleterious cardiac effects (28). In our patient, as underlying atrial flutter and heart block had been treated and initial AIT subtype was unclear, we chose to discontinue amiodarone with close monitoring for recurrence of arrythmia.

\section{CONCLUSION}

AIT is often an insidious condition which is nonetheless important to urgently recognize and treat. In clinical practice, subtype determination is often challenging and might only be possible retrospectively, limiting the application of subtypedriven treatment. Physicians should recognize the need for urgent empirical treatment in patients with cardiac disease and establish close monitoring for complications of highdose thionamides and corticosteroids. Where there are complications to first-line treatment, the expedient use of alternative treatment options is essential. This was observed 
TABLE 3 | Features of AIT Type 1 and 2 in our patient.

\begin{tabular}{|c|c|c|}
\hline & AlT Type 1 & AIT Type 2 \\
\hline Gender & & $+\left(\right.$ Male $\left.^{9}\right)$ \\
\hline Underlying thyroid disease & & $+[\operatorname{No}(1,3,9)]$ \\
\hline Frequency & & + [Much more common (14)] \\
\hline Geographical location & & + [Singapore is an iodine-sufficient region (15)] \\
\hline $\begin{array}{l}\text { Duration of amiodarone exposure prior to } \\
\text { development of thyrotoxicosis }\end{array}$ & & + [32 months of preceding amiodarone therapy (16)] \\
\hline Thyroid hormone levels & & $+[$ Higher (17)] \\
\hline Thyroid autoantibodies & & $+[$ Absent (18)] \\
\hline Thyroid ultrasound & $\begin{array}{l}+ \text { [Mildly enlarged thyroid lobes with nodules } \\
\text { up to } 1 \mathrm{~cm}(5,6)]\end{array}$ & \\
\hline Color flow Doppler & $+[$ Patchy parenchymal flow (6)] & \\
\hline Tc-99 $m$ thyroid scan & & + [Decreased uptake (5)] \\
\hline I-131 thyroid scan & & + [Decreased uptake (6)] \\
\hline Duration of hyperthyroidism & & + [Euthyroid within 3-5 months of amiodarone withdrawal (3)] \\
\hline Response to treatment & 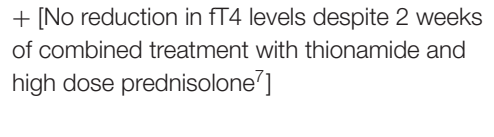 & $\begin{array}{l}+ \text { [Subsequent marked reduction in fT4 levels with } \\
\text { reintroduction of corticosteroids (19)] } \\
+ \text { [No significant response to } 5 \text { weeks of high-dose } \\
\text { thionamides] }\end{array}$ \\
\hline $\begin{array}{l}\text { Spontaneous remission } \\
\text { Require subsequent therapy for thyroid disease }\end{array}$ & & $\begin{array}{l}+ \text { [Developed euthyroidism without need for any definitive or } \\
\text { maintenance anti-thyroid treatment (1)] }\end{array}$ \\
\hline
\end{tabular}

in this case - successful treatment of AIT2 with lithium and cholestyramine.

\section{DATA AVAILABILITY STATEMENT}

The original contributions presented in the study are included in the article/supplementary material, further inquiries can be directed to the corresponding author/s.

\section{ETHICS STATEMENT}

Written informed consent was obtained from the individual(s) for the publication of any potentially identifiable images or data included in this article.

\section{AUTHOR CONTRIBUTIONS}

MC and SM made substantial contributions to the conception and design of this manuscript. MC and SM worked on the initial drafts and subsequently revised it critically for important intellectual content. MC and SM have viewed and are in approval of the final version of the manuscript to be published. MC and SM agree to be accountable for all aspects of the work. All authors contributed to the article and approved the submitted version.

\section{REFERENCES}

1. Bogazzi F, Bartalena L, Martino E. Approach to the patient with amiodarone-induced thyrotoxicosis. JClin Endocrinol Metab. (2010) 95:252935. doi: 10.1210/jc.2010-0180

2. Kurnik D, Loebstein R, Farfel Z, Ezra D, Halkin H, Olchovsky D. Complex drug-drug-disease interactions between amiodarone, warfarin, and the thyroid gland. Medicine. (2004) 83:10713. doi: 10.1097/01.md.0000123095.65294.34

3. Narayana SK, Woods DR, Boos CJ. Management of amiodaronerelated thyroid problems. Ther Adv Endocrinol Metab. (2011) 2:115-26. doi: 10.1177/2042018811398516

4. Conen D, Melly L, Kaufmann C, Bilz S, Ammann P, Schaer B, et al. Amiodarone-induced thyrotoxicosis: clinical course and predictors of outcome. J Am Coll Cardiol. (2007) 49:2350-5. doi: 10.1016/j.jacc.2007.02.054

5. Wong R, Cheung W, Stockigt JR, Topliss DJ. Heterogeneity of amiodarone-induced thyrotoxicosis: evaluation of colour-flow doppler sonography in predicting therapeutic response. Intern Med J. (2003) 33:420-6. doi: 10.1046/j.1445-5994.2003.00463.x

6. Bogazzi F, Bartalena L, Brogioni S, Mazzeo S, Vitti P, Burelli A, et al. Color flow Doppler sonography rapidly differentiates type I and type II amiodaroneinduced thyrotoxicosis. Thyroid. (1997) 7:541-5. doi: 10.1089/thy.1997.7.541

7. Ross DS, Burch HB, Cooper DS, Greenlee MC, Laurberg P, Maia AL, et al. 2016 American thyroid association guidelines for diagnosis management of hyperthyroidism other causes of thyrotoxicosis. Thyroid. (2016) 26:1343421. doi: 10.1089/thy.2016.0229

8. Gitlin M. Lithium side effects and toxicity: prevalence and management strategies. Int J Bipolar Disord. (2016) 4:27. doi: 10.1186/s40345-016-0068-y

9. Martino E, Bartalena L, Bogazzi F, Braverman LE. The effects of amiodarone on the thyroid. Endocr Rev. (2001) 22:240-54. doi: 10.1210/edrv.22.2.0427

10. Bartalena L, Bogazzi F, Chiovato L, Hubalewska-Dydejczyk A, Links TP, Vanderpump M. 2018 European Thyroid Association (ETA) guidelines for the management of amiodarone-associated thyroid dysfunction. Eur Thyroid J. (2018) 7:55-66. doi: 10.1159/000486957

11. Bartalena L, Brogioni S, Grasso L, Bogazzi F, Burelli A, Martino E. Treatment of amiodarone-induced thyrotoxicosis, a difficult challenge: results of a prospective study. J Clin Endocrinol Metab. (1996) 81:29303. doi: $10.1210 /$ jcem.81.8.8768854 
12. Tsang W, Houlden RL. Amiodarone-induced thyrotoxicosis: a review. Can J Cardiol. (2009) 25:421-4. doi: 10.1016/S0828-282X(09)70512-4

13. Bogazzi F, Bartalena L, Tomisti L, Rossi G, Tanda ML, Dell'Unto E, et al. Glucocorticoid response in amiodarone-induced thyrotoxicosis resulting from destructive thyroiditis is predicted by thyroid volume and serum free thyroid hormone concentrations. J Clin Endocrinol Metab. (2007) 92:55662. doi: 10.1210/jc.2006-2059

14. Bogazzi F, Bartalena L, Dell'Unto E, Tomisti L, Rossi G, Pepe P, et al. Proportion of type 1 and type 2 amiodarone-induced thyrotoxicosis has changed over a 27-year period in Italy. Clin Endocrinol. (2007) 67:5337. doi: 10.1111/j.1365-2265.2007.02920.x

15. Pearce EN, Farwell AP, Braverman LE. Thyroiditis. N Engl J Med. (2003) 348:2646-55. doi: 10.1056/NEJMra021194

16. Tomisti L, Rossi G, Bartalena L, Martino E, Bogazzi F. The onset time of amiodarone-induced thyrotoxicosis (AIT) depends on AIT type. Eur J Endocrinol. (2014) 171:363-8. doi: 10.1530/EJE-14-0267

17. Eaton SE, Euinton HA, Newman CM, Weetman AP, Bennet WM. Clinical experience of amiodarone-induced thyrotoxicosis over a 3-year period: role of colour-flow Doppler sonography. Clin Endocrinol. (2002) 56:338. doi: 10.1046/j.0300-0664.2001.01457.x

18. Daniels GH. Amiodarone-induced thyrotoxicosis. J Clin Endocrinol Metab. (2001) 86:3-8. doi: 10.1210/jcem.86.1.7119

19. Han TS, Williams GR, Vanderpump MPJ. Benzofuran derivatives and the thyroid. Clin Endocrinol. (2009) 70:213. doi: 10.1111/j.1365-2265.2008.03350.x

20. Kotwal A, Clark J, Lyden M, McKenzie T, Thompson G, Stan MN. Thyroidectomy for amiodarone-induced thyrotoxicosis: mayo clinic experience. J Endocr Soc. (2018) 2:1226-35. doi: 10.1210/js.2018-00259

21. Kaderli RM, Fahrner R, Christ ER, Stettler C, Fuhrer J, Martinelli $\mathrm{M}$, et al. Total thyroidectomy for amiodarone-induced thyrotoxicosis in the hyperthyroid state. Exp Clin Endocrinol Diabetes. (2016) 124:458. doi: 10.1055/s-0035-1565094

22. Dickstein G, Shechner C, Adawi F, Kaplan J, Baron E, Ish-Shalom S. Lithium treatment in amiodarone-induced thyrotoxicosis. Am J Med. (1997) 102:4548. doi: 10.1016/S0002-9343(97)00047-8
23. Ha J, Jo K, Kang B, Kim MH, Lim DJ. cholestyramine use for rapid reversion to euthyroid states in patients with thyrotoxicosis. Endocrinol Metab. (2016) 31:476. doi: 10.3803/EnM.2016.31.3.476

24. Claxton S, Sinha SN, Donovan S, Greenaway TM, Hoffman L, Loughhead $\mathrm{M}$, et al. Refractory amiodarone-associated thyrotoxicosis: an indication for thyroidectomy. ANZ J Surg. (2000) 70:174-8. doi: 10.1046/j.1440-1622.2000.01780.x

25. Bogazzi F, Bartalena L, Gasperi M, Braverman LE, Martino E. The various effects of amiodarone on thyroid function. Thyroid. (2001) 11:511-9. doi: 10.1089/1050725013 00176471

26. Uzan L, Guignat L, Meune C, Mouly S, Weber S, Bertagna X, et al. Continuation of amiodarone therapy despite type II amiodarone-induced thyrotoxicosis. Drug Saf. (2006) 29:231-6. doi: 10.2165/00002018-20062903000006

27. Eskes SA, Endert E, Fliers E, Geskus RB, Dullaart RP, Links TP, et al. Treatment of amiodarone-induced thyrotoxicosis type 2: a randomized clinical trial. J Clin Endocrinol Metab. (2012) 97:499-506. doi: 10.1210/jc.20112390

28. Bogazzi F, Bartalena L, Tomisti L, Rossi G, Brogioni S, Martino E. Continuation of amiodarone delays restoration of euthyroidism in patients with type 2 amiodarone-induced thyrotoxicosis treated with prednisone: a pilot study. J Clin Endocrinol Metab. (2011) 96:337480. doi: 10.1210/jc.2011-1678

Conflict of Interest: The authors declare that the research was conducted in the absence of any commercial or financial relationships that could be construed as a potential conflict of interest.

Copyright (c) 2020 Chua and Mok. This is an open-access article distributed under the terms of the Creative Commons Attribution License (CC BY). The use, distribution or reproduction in other forums is permitted, provided the original author(s) and the copyright owner(s) are credited and that the original publication in this journal is cited, in accordance with accepted academic practice. No use, distribution or reproduction is permitted which does not comply with these terms. 